\title{
Plasma oxytocin is related to lower cardiovascular and sympathetic reactivity to stress
}

\author{
Karen M. Grewen ${ }^{*}$ and Kathleen C. Light ${ }^{1}$ \\ University of North Carolina School of Medicine, Department of Psychiatry, CB\#: 7175, Chapel \\ Hill, NC 27599-7175, USA
}

\begin{abstract}
In addition to known reproductive and social affiliation functions, oxytocin (OT) has been identified as a cardiovascular hormone. OT synthesis and receptors are found in cardiac and vascular tissue. Animal studies suggest that OT activates an 'anti-stress' response that reduces cardiovascular and neuroendocrine stress reactivity. We tested 28 early postpartum mothers, obtaining multiple blood samples for OT, the sympathetic marker, norepinephrine (NE), and the lactation hormone, prolactin, while monitoring their cardiovascular responses to two stressors: public speaking and forehead cold pressor. Although plasma OT did not increase reliably from pre-stress levels during stressors, greater overall OT level was related to greater vasodilation and cardiac stroke volume responses to both tasks, to reduction in heart rate to the cold pressor, as well as to lower plasma NE and higher prolactin levels. In contrast, higher NE was linked to increases in heart rate and decreases in stroke volume. These data support a cardioprotective role for OT, which may influence the magnitude and hemodynamic determinants of cardiovascular stress responses.
\end{abstract}

\section{Keywords}

Oxytocin; Norepinephrine; Cardiovascular stress response; Vascular resistance; Heart rate; Cardiac output

\section{Introduction}

\subsection{Oxytocin is a cardiovascular hormone}

In the past decade substantial attention has been focused on the role of the neuropeptide, oxytocin (OT), in social behaviors. However, OT has also been identified as a cardiovascular hormone (Petersson, 2002). OT synthesis and receptors are reported in both cardiac and vascular tissue in non-human mammalian species (Gutkowska et al., 2000; Jankowski et al., 1998, 2000), and OT acts both centrally and peripherally at multiple sites including brainstem, heart, and vessels to exert acute and long-term inhibitory effects on cardiovascular activity (Meisenbert and Simmons, 1983; Nakamura et al., 2000; Petersson et al., 1996, 1997, 1999a,b; Sofroniew, 1983; Stock and Uvnas-Moberg, 1988; Thibonnier et al., 1999a,b). In animal models, daily peripheral administration of exogenous OT for 5 days

\footnotetext{
(C) 2011 Elsevier B.V. All rights reserved.

*Corresponding author. Tel.: +1 919966 2544; fax: +1 919966 0708. karen grewen@med.unc.edu (K.M. Grewen), Kathleen.C.Light@hsc.utah.edu (K.C. Light)..

${ }^{1}$ Present address: University of Utah School of Medicine, Department of Anesthesia, Salt Lake City, UT 84112, USA

Conflict of interest

The authors have no conflicts of interest to disclose.
} 
leads to blood pressure (BP) decreases lasting 2 months or more (Holst et al., 2002; Petersson and Uvnas-Moberg, 2008). Similarly, OT administered in vitro reduces the rate and force of cardiac cells' intrinsic contractions causing them to 'beat' more slowly and contract less forcefully (Mukaddam-Daher et al., 2001). In humans, studies reporting on BP effects of chronic OT administration are lacking. However short term intravenous (IV) administration of OT to women to enhance uterine contractions or decrease blood loss during labor or caesarean delivery confirm its effect in decreasing blood pressure (Sartain et al., 2008; Simpson and Knox, 2009; Thomas et al., 2007). This hypotensive response to OT is due to decreases in total vascular resistance despite compensatory increases in heart rate, stroke volume and cardiac output. In fact, even though specific organs and tissues may show local vasoconstriction with OT administration, decreases in total vascular resistance and BP that are potentially life threatening can occur (Archer et al., 2008).

\subsection{Oxytocin and social interactions both influence cardiovascular function}

Social behaviors are related to both OT and cardiovascular activity, and OT may serve as an important physiological mediator of the cardioprotective benefit of social bonding (Knox and Uvnas-Moberg, 1998). Animal studies reveal that affiliative social interactions elicit increases in OT activity, which then activate and integrate an 'anti-stress' response that promotes bonding, relaxation and growth, while reducing cardiovascular and neuroendocrine stress responsivity (Callahan et al., 1989; Petersson and Uvnas-Moberg, 2007, 2008; Uvnas-Moberg, 1998; Uvnas-Moberg et al., 2001; Uvnas-Moberg and Petersson, 2004; Wsol et al., 2008). In rats, daily ventral stroking for 5 days is linked to long-lasting BP decreases very much like those induced by exogenous OT administration, and it is presumed that this effect is due to increases in endogenous OT activity (Holst et al., 2002). In female prairie voles, social isolation (considered a stressor), results in higher basal heart rate (HR) levels and decreased HR variability, and these effects are reversed with subcutaneous OT administration (Grippo et al., 2009). In humans, a number of studies have linked higher plasma OT to lower BP. We observed this in early postpartum mothers both on days when they had recent infant contact and when they did not, and in married women studied after 10 min of structured warm contact with their husbands (Grewen et al., 2005; Light et al., 2000, 2005a,b). In addition to lower BP in laboratory studies, higher OT responses to structured affectionate interaction with infants were associated with lower maternal 24-h ambulatory BP levels at home (Light et al., 2000, 2004). We also reported consistent inverse associations between plasma OT and the sympathetic biomarker, norepinephrine (NE), in women during rest and in response to pleasant partner contact (Grewen et al., 2005), but we have not measured these hormones simultaneously during stress. Similarly, a month-long intervention in couples, involving caring support through Rosen listening touch, a therapeutic method using light touch to communicate acceptance, empathy and caring and to sense subtle physical and emotional responses felt by another (Rosen, 2003), resulted in increases in salivary OT and decreases in the indirect sympathetic marker, salivary alpha-amylase, in both husbands and wives. In addition, in husbands but not wives, 24-h BP was also reduced. However, since the wives had lower BP prior to the intervention, this may have been due to a 'floor effect' (Holt-Lunstad et al., 2008).

\subsection{Oxytocin may attenuate physiological response to stress}

Although less studied than the OT-social affiliation links, findings from both animal and human studies suggest that plasma OT may reduce physiologic stress responses. OT knockout mice exhibit greater BP and corticosterone responses to acute and chronic stress (Bernatova et al., 2004; Michelini et al., 2003). In rats, blocking central OT with intracerebral infusion of OT antagonist enhances HR and BP responses to acute stress but does not effect resting levels (Wsol et al., 2008). Findings from human studies are mixed, however. This may be due to differences in the kinds of stressors used and/or in the subject 
populations being studied. Light et al. (2005a,a) reported that in postmenopausal women participating in a hormone replacement therapy (HRT) trial, greater treatment-induced increases in oxytocinergic activity (indexed by plasma levels of an OT precursor) were associated with greater post-treatment reductions in BP and vascular resistance reactivity to a battery of experimental stressors including speech, stroop and cold pressor tasks. More recently, Taylor et al. (2006) reported that although postmenopausal women self-selected for HRT had higher plasma OT compared with non-treated women, higher OT was not related to blood pressure or heart rate reactivity to the Trier Social Stress Test (TSST). Similarly, Altemus et al. (2001) reported that in postpartum lactating and non-lactating women and reproductiveaged control women, OT levels were not associated with blood pressure or heart rate reactivity to the TSST, but lactation was related to greater parasympathetic cardiac control.

\section{The present study}

In the current study we investigated how circulating OT may be related to cardiovascular and sympathetic nervous system responses to stress in women tested between 2 and 6 months postpartum. We measured BP and HR, as well as the hemodynamic determinants of BP, cardiac output and vascular resistance. Although prior studies have sometimes, but not always, shown relationships between OT and BP responses to stressors in postpartum women (Altemus et al., 2001), we are not aware of any prior study that has examined whether higher endogenous levels of plasma OT may be reliably associated with altered cardiac or vascular resistance responses to stress. Because both the heart and the vasculature have OT receptors (Gutkowska et al., 2000), decreases in BP associated with higher circulating OT may be related to either decreased vascular resistance, decreased cardiac rate and/or contractile force (as reflected in cardiac output and Heather index), or a combination thereof. We recruited 28 mothers of infants, and repeatedly obtained blood samples for OT while monitoring their cardiovascular responses to a stressful speech task followed by the forehead cold pressor test. Based on our prior research, we hypothesized that mothers who showed higher overall levels of plasma OT (reflecting higher overall oxytocinergic activity) would demonstrate lesser vasoconstriction response to one or both of these stressors, and lower levels of plasma norepinephrine, the primary sympathetic nervous system biomarker mediating increases in heart rate and alpha-adrenergic vasoconstriction. We also assessed another lactation hormone, serum prolactin, to serve as a validation of the OT measurement. We expected strong positive OT-prolactin correlations, since simultaneous OT and prolactin increases are necessary to accomplish lactation. Consistent with our previous mother-infant protocols, we measured maternal stress reactivity following a 10-min affectionate motherinfant interaction. Because of the pulsatile release of OT, and because of our interest in how hormone levels influence cardiovascular responses over time, Area Under the Curve (AUC) values were calculated for each of the three hormones measured: oxytocin (OT), norepinephrine (NE), and prolactin (PRL). Using this method, two values are created for each hormone: an unadjusted AUC measure $\left(\mathrm{AUC}_{\mathrm{G}}=\right.$ "AUC with respect to Ground"), and a measure adjusted for differences in baseline level $\left(\mathrm{AUC}_{\mathrm{I}}=\right.$ "AUC with respect to Increase"). $\mathrm{AUC}_{\mathrm{I}}$ is described as a measure of reactivity, while $\mathrm{AUC}_{\mathrm{G}}$ is described as an index of overall hormone activity across the protocol (Pruessner et al., 2003). Prior work has not addressed whether overall levels or reactivity to stressors are better indices of OT effects on cardiovascular function. Therefore, we examined both measures to better understand the cardiovascular-OT relationship. 


\section{Method}

\subsection{Participants}

Twenty-eight healthy mothers were recruited from the community during the perinatal period for testing within the window of 2-6 months postpartum. Of these, 18 breastfed exclusively from birth, 5 formula-fed exclusively, and 5 endorsed combined breast and formula-feeding. Three subjects were cigarette smokers ( 2 exclusive formula, 1 exclusive breast). Exclusion criteria included self-report at telephone screening interview of prescription or nonprescription drug use, recent history of major depressive disorder and other psychological disorders, history of chronic disease in mother or infant, multiple birth, and low birth weight (less than $2500 \mathrm{~g}$ ).

\subsection{Protocol}

All procedures were approved by the university institutional review board and all participants underwent informed consent before testing. Mothers were instrumented with cardiovascular electrodes for measurement of cardiovascular activity and an intravenous catheter was then inserted into an antecubital vein, with normal saline drip set at $0.25-1.00$ drops $\mathrm{s}^{-1}$ to maintain lumen patency. The protocol consisted of the following events in fixed order: instrumentation, habituation (10 $\mathrm{min})$, baseline rest $(6 \mathrm{~min})$, mother-infant interaction (10 min), speech task (7 min), forehead cold pressor $(2.5 \mathrm{~min})$, post-stress recovery $(6 \mathrm{~min})$. The first blood draw was done at least $1 \mathrm{~h}$ after the last infant feeding. Infants were present in the room for the entire protocol, cared for by a female research assistant. Mothers were allowed to direct care throughout, and to talk to and touch their infants ad libitum during segments when measurements were not being taken. On a separate day, mothers completed questionnaires at home including the Edinburgh Postnatal Depression Scale and the Spielberger Trait Anxiety Inventory, and an in-house survey of health and socioeconomic information.

\subsection{Experimental events}

Baseline rest (BAS) and post-stress recovery (REC)—Subjects sat quietly for 6 min. Mother-infant interaction (INTXN): Mothers held their infants on their laps or next to them on a love seat, and interacted with them ad libitum as they watched a musical video about babies for $9.5 \mathrm{~min}$, and then engaged in a 20 -s face-to-face hug at the end of the period. No cardiovascular or endocrine measures were taken during this time due to variability in movement, effort and speaking, however one cardiovascular reading and blood draw were done following this period after mother sat quietly for approximately $1 \mathrm{~min}$.

Speech task-Prior to attending the session, mothers were required to list brief descriptions of three recent interpersonal situations in which they felt angry or stressed (i.e., 'argument with my husband about helping me with the baby'). Immediately prior to the speech task, the experimenter informed the subject which stressful event she would be speaking about. She was given 1 min to silently prepare her talk (PRP), and 3 min to give her audio-taped speech (SPC). The tape was immediately rewound and the subject was then asked to listen to the replay of her speech (REP).

Forehead cold pressor (ICE)—A plastic bag of ice and water $\left(\sim{ }^{\circ} \mathrm{C}\right)$ was held on the forehead for $2.5 \mathrm{~min}$. Subjects were required to rate their forehead pain shortly before and at the end of this task, immediately prior to removal of ice from the forehead. This task causes pain, similar to an 'ice cream headache,' and reliably elicits alpha-adrenergically mediated vasoconstriction in most subjects. 


\subsection{Venous blood samples}

Venous blood was collected into pre-chilled vacutainer tubes, immediately cold-centrigued, aliquoted into pre-chilled cryotubes, and stored at $-80{ }^{\circ} \mathrm{C}$ for later endocrine assays.

Oxytocin: nine plasma samples were collected into heparin-treated tubes at the following time points: Post-INTXN, SPC (2:00, 3:00), REP (2:00, 3:00), ICE (1:00, 2:00), and REC (4:00 and 5:00). Efforts were made to sample blood for OT assay twice during each stress and recovery periods, drawing samples as close to $1 \mathrm{~min}$ apart as possible, in order to capture OT variation due to pulsatile release. The level of OT in plasma was determined by extraction and radioimmunoassay (RIA) in the laboratory of Janet Amico (Amico, Seif and Robinson, 1981; Amico and Hempel, 1990). The intra-assay coefficient of variation was 10$12 \%$. The lower limit of sensitivity was $0.5 \mathrm{pg} / \mathrm{ml}$ and $14.8 \%$ of samples fell at or below the lower limit of detection. Plasma OT values ranged from $0.5 \mathrm{pg} / \mathrm{ml}$ to $6.8 \mathrm{pg} / \mathrm{ml}$.

Norepinephrine (NE) and Prolactin (PRL) samples were collected once during each experimental event, at the following times: (Post-INTXN, SPC 2:30, REP 2:30, ICE 2:00, and REC 5:30). NE in heparin-treated plasma was measured with reverse-phase high performance liquid chromatography (HPLC) at the UNC GCRC Core laboratory (lower limit sensitivity $=5 \mathrm{pg} / \mathrm{ml}$, inter- and intra-assay CV $<10 \%$ ). Serum PRL levels were assessed using a commercially available RIA (Becton Dickenson, Orangeburg, NY; sensitivity $=1.0 \mathrm{ng} / \mathrm{m}$; intra- and inter-assay CV 5\% and 9\%, respectively).

\subsection{Cardiovascular assessments}

Blood pressure (BP) was assessed with the Suntech 4240 Exercise Blood Pressure monitor (Suntech, Raleigh, NC), a device whose prototype has been validated against direct arterial and standard auscultatory measurements. Impedance cardiography was used to estimate stroke volume (Minnesota Impedance Cardiograph, model 304B, Surcom, Minneapolis, $\mathrm{MN}$ ), along with software designed to calculate hemodynamics and systolic time intervals (COPWIN, Microtronics, Chapel Hill, NC). A tetrapolar band electrode configuration was used to determine the change in electrical impedance to a low-voltage signal that occurs with, and is proportional to, changes in blood flow in the ascending and descending aorta with each cardiac contraction. Stroke volume (SV, measured as $\mathrm{mL}$ per cardiac cycle) was estimated from cardiac cycles ensemble-averaged over $40 \mathrm{~s}$ epochs, based on the Kubicek equation (Kubicek et al., 1970). Skin was prepared by cleaning with alcohol before applying 3 ECG spot electrodes (ConMed Invisatrace). A standard 3-lead ECG bipolar Lead I configuration was used to derive heart rate from the peak $R$-wave intervals $(1000 \mathrm{~Hz}$ sampling rate). Cardiac time intervals were calculated using ECG and impedance waveforms. ECG and impedance artifacts were detected by the COPWIN software and manual inspection of each ensemble average as per criteria described by Sherwood et al. (1990a,b). Mean arterial pressure, derived from BP readings taken concurrently with each ensemble-averaged measure of cardiac output (measured in $\mathrm{L} / \mathrm{min}$ ), was used to determine total peripheral vascular resistance. In order to control for differences in body size and obesity, stroke volume, cardiac output and total peripheral resistance were indexed for body surface area (BSA) as per the method described by Sherwood et al. (1990a,b) and are hence referred to as stroke volume index (SVI), cardiac index (CI), and total systemic vascular resistance index (VRI). Pre-ejection period (PEP), a systolic time interval determined by the ECG and impedance cardiogram, is defined as the time (ms) between ECG Q wave and dZ/ $\mathrm{d} t \mathrm{~B}$ point, and is used as an index of myocardial contractility, and sympathetic nervous system influence on the heart (Sherwood et al., 1990a). Heather Index (HI: $\Omega / \mathrm{s}^{2}$ ), a measure corresponding roughly to changes in cardiac contractility, was also determined by the COPWIN software and is defined as a ratio of $-\mathrm{d} Z / \mathrm{d} t_{\max }$ to the interval from that point to the $R$-wave peak (Hill and Merrifield, 1976; Mezzacappa et al., 1999). These cardiovascular 
measures were assessed at the onset of each of the following minutes during each experimental condition: baseline (BAS): 1:00, 3:00, 5:00; interaction (INTXN): 10:00, speech preparation (PRP): 0:00; speech (SPC): 1:00, 2:00, 3:00; replay (REP): 1:00, 3:00; cold pressor (ICE): 0:00, 1:00, 2:00; recovery (REC): 1:00, 3:00, 5:00.

\subsection{Stress and pain measures}

Three identical Visual Analog Scales (VAS) were used to assess subjects' self-reported stress immediately following baseline, speech task, and cold pressor $(0=$ no stress/anxiety at all, $10=$ the most intense stress/anxiety imaginable). Forehead pain intensity was reported twice: following speech replay and immediately prior to ice removal at the end of cold pressor (Minute 2:15), using two identical VASs $(0=$ no head pain at all, $10=$ the most intense head pain imaginable).

\subsection{Statistical analysis}

$\mathrm{AUC}_{\mathrm{G}}$ and $\mathrm{AUC}_{\mathrm{I}}$ values for all three hormones were calculated as per the method described by Pruessner (Pruessner et al., 2003), log-transformed to correct for non-Gaussian distribution, and then correlated with each other and with other physiological measures using Pearson product moment correlations $(r)$. Means and standard errors calculated for all eight cardiovascular variables (SBP, DBP, HR, CI, SVI, HI, PEP, VRI) at each of the 16 measurement time points are depicted in Fig. 1. Each subject's mean value for each of the 8 cardiovascular variables was then calculated within each experimental condition in which multiple readings were done (baseline, speech, replay, cold pressor and recovery). Cardiovascular reactivity to infant interaction values were calculated as (INTXN - mean baseline value). Cardio-vascular stress reactivity values for speech and cold pressor tasks were calculated as change scores (Task Minute 1 - mean baseline value) during each of the two stressors. Minute 1 was chosen because peak cardiovascular values were seen at that time during both speech and cold pressor. Paired $t$-tests were used to examine differences in stress levels compared with mean baseline values, and differences between infant interaction and baseline values. Pearson correlations were used to test associations between hormone AUC values, and between cardiovascular reactivity (change scores) and hormone values. Significant correlations were furthered examined using simultaneous regression controlling for covariates with possible influence on reactivity. Models of blood pressure, heart rate, pre-ejection period and Heather index were adjusted for smoking ( 3 smokers, 25 nonsmokers), feeding method (18 breast, 10 formula/combo), BMI and baseline value. Models of stroke volume index (SVI), vascular resistance index (VRI) and cardiac index (CI) were adjusted for smoking, feeding and baseline value. Because these variables are already adjusted for body surface area (BSA), which was strongly correlated with BMI ( $r=$ 96, $p<0.0001$ ), analyses of VRI, CI and SVI were not adjusted for BMI. Because OT $\mathrm{AUC}_{\mathrm{G}}$ and VAS rating of post-speech stress were both related to lower VRI and SVI responses to speech, stress ratings were added to these models. Similarly, in cases where cardiovascular reactivity was correlated with both NE and OT, regression analyses were performed to test variance in reactivity (change scores) attributable to OT $\mathrm{AUC}_{\mathrm{G}}$ and $\mathrm{NE}$ $\mathrm{AUC}_{\mathrm{G}}$ when both were present in the model.

\section{Results}

\subsection{Subject characteristics}

Subject characteristics are provided in Table 1 . The majority of mothers were white (4 African-American, 22 Caucasian, 2 Hispanic), with at least a 4-year college degree ( $8=$ less than 4-year degree, $16=4$-year degree, $8=$ post-graduate education). Infant postnatal age ranged from 2.3 to 6.3 months at time of testing, while maternal age ranged from 24 to 41 years. All subjects were normotensive. The sample included 18 mothers who exclusively 
breast-fed and 10 mothers who used formula feeding in whole $(n=5)$ or in combination with breast feeding $(n=5)$. In addition to mean totals across the 28 subjects, Table 1 also shows mean values separately for exclusive breasting feeding and formula-feeding (exclusive and partial combined) subjects. Exclusive breast-feeders had lower BMI, greater stress ratings, and marginally lower plasma norepinephrine calculated as area under the curve across the session. When Exclusive Formula-feeding mothers $(n=5)$ were examined separately, they had significantly higher plasma norepinephrine than exclusive breastfeeders (NE $\mathrm{AUC}_{\mathrm{G}}$ means: 966.5 vs. $634.7 \mathrm{ng} / \mathrm{mL} ; F=6.01, p=0.042$ ) but did not differ on oxytocin or any other variable listed in Table 1. Prolactin data was available for only one exclusive formula feeding mother therefore comparisons could not be made between exclusive breast vs. exclusive formula for prolactin levels.

\subsection{Cardiovascular responses to stressors}

Fig. 1 depicts subjects' cardiovascular values measured at each of the 16 sampling times during the protocol. Speech: The speech task induced significant increases in SBP, DBP and HR compared with mean baseline levels (paired $t$-test $p$ values $<0.05$ ). Cold pressor induced significant increases in SBP, DBP, and VRI, and significant decreases in HR. Cardiac output (CI) and contractility, quantified as heather index, were immediately but transiently reduced below baseline levels at Minute $0(p<0.01)$. Significant increases in both SBP and DBP were seen at Minute 1 during each of the 2 stressors while peak vascular resistance levels occurred only during the forehead cold pressor task. Stroke volume and pre-ejection period were not significantly different during stress compared with baseline. Reactivity of stroke volume, pre-ejection period and vascular resistance was inconsistent across subjects, as reflected in higher variability in these measures compared with lower variation in blood pressure and heart rate across subjects at each time point. Table 2 lists delta scores (change from baseline to Minute 1) for cardiovascular values (mean \pm standard error) during each of the two stressors, speech and cold pressor.

\subsection{Cardiovascular response to infant interaction}

Movement-related artifacts or disconnection of impedance sensors during mother-infant interaction resulted in lost data for impedance-derived values in 11 subjects (PEP, SVI, CI, VRI, HI) at the single reading following this period. However SBP, DBP and HR were available for all 28 subjects, and change from baseline levels in SBP, DBP and HR did not differ in those with $(n=17)$ and without $(n=11)$ available impedance data. Compared with baseline, $\operatorname{SBP}(t=3.95, p=0.0005)$, HR $(t=2.07, p=0.045)$, stroke volume (SVI: $t=2.60$, $p=0.018)$ and cardiac output (CI: $t=3.09, p=0.0071)$ were significantly higher following infant interaction. Pre-ejection period (PEP: $t=-3.55, p=0.0023$ ) was significantly reduced from baseline levels, consistent with increased HR. Vascular resistance was marginally decreased (VRI: $t=-2.01, p=0.06$ ). This pattern of increased blood pressure, heart rate, cardiac output and mild vasodilation may have been due to the recent physical exertion of holding, talking to and playing with the infant.

\subsection{Oxytocin, norepinephrine, and prolactin responses and associations}

Mean plasma OT levels at all 9 sampling time points, and mean plasma norepinephrine (NE) and serum prolactin (PRL) levels, sampled 5 times during the protocol, are depicted in Fig. $2 \mathrm{~A}-\mathrm{C}$ respectively. OT data were available for all 28 subjects. NE data for 26 , and PRL data for 20 subjects were also available. Plasma NE increased significantly over baseline levels during the cold pressor task (paired $t$-test $t=2.81, p=0.0096$ ). There was no significant increase in OT or PRL in response to stress, and substantial variability in OT and PRL at each time point. Total plasma OT was inversely correlated with norepinephrine when each was calculated as $\mathrm{AUC}_{\mathrm{G}}(r=-0.40 p=0.05$, depicted in Fig. 2D), or when each was calculated as $\mathrm{AUC}_{\mathrm{I}}(r=-0.41, p=0.04)$. Total serum prolactin $\mathrm{AUC}_{\mathrm{G}}$, sampled at the same 
five time points as plasma NE, was also correlated with OT $\mathrm{AUC}_{\mathrm{G}}(r=+0.50, p=0.02)$ and inversely correlated with $\mathrm{NE} \mathrm{AUC}_{\mathrm{G}}(r=-0.49, p=0.03)$. In response to speech stressor, 11 women demonstrated oxytocin increases above baseline, while 9 showed no change and 8 had decreased levels. There were 6 exclusive breast feeders in each of these groups. Similar differences in OT response to cold pressor were observed (12 increased, 6 no change, 10 decreased).

\subsubsection{Oxytocin, Norepinephrine and Prolactin based on infant feeding status} -When OT was examined separately for breast and formula feeding groups, there was no significant difference in OT level or change in OT at any time point (Fig. 2E). Fig. 2F shows that plasma NE was marginally greater in formula feeders compared with exclusive breast feeders during speech task $(232.1 \pm 23.29$ vs. $177.9 \pm 16.5 \mathrm{pg} / \mathrm{mL} ; t=1.93, p<0.07)$. Serum prolactin levels, shown in Fig. 2G, did not change significantly across the protocol in breast or formula feeders.

\subsection{Behavioral responses}

Self-reported stress and pain, rated on visual analog scales, are listed in Table 1. Stress ratings increased above baseline when rated immediately following speech $(t=4.84, p<$ $0.0001)$ and cold pressor $(t=2.67, p=0.013)$, indicating that these tasks were experienced as stressors. Breast feeding women reported greater stress at all 3 time points compared with formula feeding women, and had greater increases in stress ratings when regression models were adjusted for baseline differences (not shown). Across the full sample, greater stress during the speech was accompanied by greater vasodilation and increases in cardiac output, consistent with a 'fight-or-flight' beta-adrenergically mediated stress response. Greater stress ratings of speech task correlated with reductions in vascular resistance $(r=-0.58, p$ $<.01)$, and increases in stroke volume $(r=+0.53, p<.01)$ and cardiac output $(r=+0.69, p$ $<.0005)$ during the task. Self-reported forehead pain ratings increased after cold pressor $(t=$ $15.87, p<0.0001$ ), and were related to greater stress reported at the same time (Spearman $r$ $=+0.64, p=0.0004)$. Neither stress nor pain ratings were correlated with any individual or composite AUC hormone values.

\subsection{OT, NE and PRL are associated with cardiovascular responses to stress}

Plasma oxytocin and norepinephrine were each related to stress-induced changes in some, but not all, cardiovascular variables, and had opposing effects on cardiac responses. Table 2 summarizes Pearson correlations found between cardiovascular stress reactivity values (delta scores) and OT and NE calculated as both total hormone $\left(\mathrm{AUC}_{\mathrm{G}}\right)$ and reactivity $\left(\mathrm{AUC}_{\mathrm{I}}\right)$ values.

4.6.1. OT and vascular resistance-Greater oxytocin levels were correlated with smaller increases (or greater decreases) in vascular resistance (delta VRI) compared to baseline in response to both the speech $(r=-0.51, p=0.007)$ and the cold pressor tasks $(r=$ $-0.38, p=0.05)$. Fig. 3 depicts these negative correlations of OT $\mathrm{AUC}_{\mathrm{G}}$ with changes in VRI during Minute 1 of the speech (Fig. 3A) and Minute 1 of cold pressor (Fig. 3B). Because greater OT and stress rating were both related to lower VRI responses to speech, we regressed VRI response on both post-speech stress rating and OT AUC $\mathrm{G}_{\mathrm{G}}$. Both OT and stress rating remained independent predictors of decreases in vascular resistance (overall $F_{2,26}=10.18, p=0.0022, R$-squared $=0.44$; OT AUC $\mathrm{G}_{\mathrm{G}}$ : beta: $-0.47, p<0.004$, stress: beta: $-0.46, p=0.005$ ). When additional covariates were added (feeding status, baseline vascular resistance, smoking status) oxytocin remained an independent predictor of vascular resistance reactivity to speech $\left(\mathrm{OT} \mathrm{AUC}_{\mathrm{G}}\right.$ beta $=-0.34, t=-2.09, p=.048$ ) and cold pressor $\left(\mathrm{OT} \mathrm{AUC}_{\mathrm{G}}\right.$ beta $\left.=-0.41, t=-2.39, p=0.026\right)$. 
4.6.2. OT, NE and cardiac responses-Table 2 shows that OT was not significantly correlated with stress-induced changes in blood pressure (BP) or cardiac output (CI). OT was, however, related to the determinants of cardiac output: heart rate and stroke volume. Greater OT correlated with greater increases in stroke volume (SVI) during both tasks, and to greater decreases in heart rate (HR) response to cold pressor. Models were then adjusted for relevant covariates. Stroke volume reactivity to speech: When stress rating of the speech task was added to the model predicting SVI response, both OT and stress remained independent predictors of greater SVI response (overall $F_{2,26}=7.098, p=0.0038, R$-squared $=0.37$; OT AUC $\mathrm{G}_{\mathrm{G}}$ beta: $+0.46, p<0.01$, stress: beta: $\left.+0.38, p<0.05\right)$. OT remained marginally predictive (beta $=+30, p=.087$ ) when baseline SVI, feeding and smoking status were added to the model. In contrast to OT, greater NE was related to greater increases in HR during both stressors, and to decreases in stroke volume (SVI) and contractility (HI) during the cold pressor. HR reactivity to cold pressor: OT and NE independently accounted for significant amounts of variance in HR reactivity to cold pressor when both were entered into the model simultaneously (overall $F_{2,26}=8.12, p=0.0022, R$-squared $=0.41$; OT $\mathrm{AUC}_{\mathrm{G}}$ : beta $=-0.42, p=0.016$; $\mathrm{NE} \mathrm{AUC}_{\mathrm{G}}$ : beta $=+0.53, p=0.003$ ) and OT remained a significant predictor in the full model (OT beta $=-0.47, p=.014$ ). Similarly, OT independently accounted for significant amounts of variance in SVI reactivity to cold pressor when both OT and NE were entered into linear regression, however NE was no longer a significant predictor of SVI change when OT was present in the model $\left(F_{2,26}=\right.$ 5.64, $p=0.01, R$-squared $=0.36$; OT beta $=+0.43, p=0.037$; NE beta $=-0.29, p=\mathrm{ns})$. OT remained a significant predictor in the full model (OT beta $=+0.47, p=0.037$ ) in which baseline SVI, feeding and smoking status were added.

4.6.3. Prolactin (PRL) —Although prolactin data was available for fewer subjects $(n=$ 20), correlations with cardiovascular reactivity values were similar to those seen with oxytocin. Total prolactin (PRL $\mathrm{AUC}_{\mathrm{G}}$ ) was significantly correlated with reductions in vascular resistance and increases in stroke volume during the speech task (delta VRI: $r=$ -0.59 ; delta SVI: $r=+0.43 ; p<0.05$ ). Similarly, PRL $\mathrm{AUC}_{\mathrm{G}}$ was correlated with reductions in vascular resistance and heart rate during the cold pressor (delta VRI: $r=-0.51$; delta HR: $r=-0.52 ; p<0.05)$. In this smaller subset of women, oxytocin was correlated with cardiovascular reactivity values in the same or greater magnitudes seen in the complete sample of 28 women (speech: delta VRI $r=-0.52$, delta SVI $r=+0.046$, cold pressor: delta VRI $r=-0.45, p<0.05$; cold pressor delta HR: $r=-0.68$, delta SVI: $r=-0.65, p<0.001$ ). PRL was also related to reduced DBP at the end of infant interaction compared with resting baseline (delta DBP: $r=-0.54 ; p<0.05$ ).

\section{Discussion}

Our prior research focused on linking higher oxytocin activity to the quality of the motherinfant relationship and the husband-wife relationship (Grewen et al., 2005; Holt-Lunstad et al., 2008; Light et al., 2005a,b). In the present investigation, we focused instead on the role of oxytocin in modulation of cardiovascular and sympathetic responses to stress. In 28 breast- and bottle-feeding mothers of infants tested in a protocol involving two behavioral stressors, blood pressure was increased in response to both tasks however changes in vascular and cardiac activity that determine blood pressure differed predictably by task characteristics. Importantly, oxytocin levels were related to attenuated cardiac and vascular reactivity during both tasks. In contrast, higher sympathetic activity, indexed by plasma norepinephrine, was related to greater heart rate (HR) increases to speech and lesser reductions during cold pressor. Higher overall circulating levels of oxytocin were associated with lower overall plasma norepinephrine (NE), and independent of NE, with smaller HR increases to speech and greater HR reductions during the cold pressor. Furthermore, overall oxytocin levels were correlated with lesser vasoconstriction during both stressors, and this 
effect was independent of infant feeding method, smoking status, baseline differences, and severity of perceived stress reported by the individual. These observations are consistent with the interpretation that, in addition to its role in reproductive functions and social affiliation, oxytocin modulates cardiovascular stress responses elicited by both active and passive stressors. This pattern further suggests that oxytocin is part of an inhibitory or antistress system designed to limit the severity and duration of stress-induced departures from cardiovascular homeostasis. One aspect of this effect involves decreases in sympathetic nervous system outflow to heart and vessels, evidenced by lower plasma NE. Other aspects that seem partly independent of the sympathetic inhibition involve blunted cardiac and vascular responses to stress, possibly resulting from peripheral binding of oxytocin to receptors on vascular and cardiac tissue.

Two other points are worth emphasizing. First, the higher OT levels were defined based on the Area Under the Curve of 9 different blood samples extending over a 30-min period. Because OT is released in a pulsatile fashion, more frequent sampling makes it more likely to include at least some of the peak levels and to represent each subject's average levels more accurately than using a single sample or a few samples obtained over a greater time period. Second, although both stressors elicit increases in blood pressure, these two challenges were selected to include one (speech) which enhances beta-adrenergic activity, and another (forehead cold pressor) in which pressor responses are accomplished via alphaadrenergic activity. The speech task often produces vasodilation or no change in vascular resistance, as well increases in cardiac output, while the cold pressor almost universally produces vasoconstriction and an initial bradycardic response (Sherwood et al., 1990a,b). Our finding that higher OT AUC is associated with lesser vascular resistance responses during both tasks suggests that OT is not solely influencing either alpha- or beta-adrenergic activity, but may be acting directly upon the vasculature. Previous studies have confirmed the presence of functional OT receptors in both vascular endothelial cells and vascular smooth muscle cells in rats (Gutkowska et al., 2000) and humans (Szeto et al., 2008; Thibonnier et al., 1999a,b), and OT synthesis has been reported in large vessels in rodent studies (Jankowski et al., 1998, 2000). One salient consequence of OT binding to OT receptors on vascular endothelium is calcium-dependent nitric oxide-mediated relaxation of vascular smooth muscle, with subsequent vasodilation. Thus, our observations indicate that human OT receptors, when activated, may have similar vasodilatory properties at the level of vascular endothelial and/or smooth muscle cells.

The associations between greater OT and lower HR to both tasks, which were only significant during the cold pressor, could be due to the decrease in sympathetic activity, to an increase in parasympathetic activity, or to OT acting directly on OT receptors in cardiac tissue. Animal and in vitro research reveal an intrinsic OT system within the heart, which synthesizes OT and expresses OT receptors. OT binding to OT-R within the heart stimulates release of atrial naturetic peptide, resulting in reduced HR, contractility and blood volume. OT-induced reductions in HR responses, allowing greater ventricular filling time, may have contributed to the observed correlations between greater OT and greater stroke volume responses to cold pressor. An alternative or additional factor may have been OT-induced reductions in VRI, resulting in smaller afterload impeding ventricular ejection.

We did not observe acute changes in OT levels in response to stressful challenges. This may have been influenced by the fact that baseline levels of OT were obtained shortly after mothers interacted with and hugged their infants, which could have stimulated oxytocin release and milk let down in some women. In addition we did not control for the exact timing between last feeding and experimental events. Nevertheless, with or without an OT increase to stress, higher OT levels at baseline and overall could still act to restrain cardiovascular reactivity. However the lack of a statistically significant OT stress response is 
relevant to a growing literature with conflicting findings. Although OT appears to be elevated or dysregulated in some chronically stressful conditions including major depressive disorder (Cyranowski et al., 2008), relationship distress (Taylor et al., 2010), interpersonal distress (Turner et al., 1999) and childhood trauma (Pierrehumbert et al., 2010), findings of OT reactivity to acute stress are mixed. Stress-induced OT elevations have not been observed in response to the Trier Social Stress Test (TSST) in postmenopausal women (Taylor et al., 2006), or in postpartum and premenopausal control women (Altemus et al., 2001; Heinrichs et al., 2001). However, recently TSST-induced increases in OT were reported in healthy adults with and without history of childhood cancer or sexual abuse, with greatest OT reactivity exhibited by those with childhood cancer (Pierrehumbert et al., 2010).

We did not observe differences in mothers' oxytocin or cardiovascular reactivity based on infant feeding method. This may be because the study was not designed to examine differences by lactation status, thus we did not include a sufficient number of exclusive formula-feeding women to adequately test differences. Our comparison of exclusive breast feeders to women who either supplemented or fed exclusively with formula may not have been ideal since women who supplement breast with formula feeding may be more like exclusive breast feeders or may be different from both exclusive breast and formula feeding groups. Notably, in the current study we observed substantial variability in OT levels during stressors in both breast- and formula-feeding subjects. This is consistent with our earlier finding of OT reactivity in postpartum women in response to speech stressor following infant contact (Light et al., 2000). In both studies, we found that OT increased in some subjects, but decreased or remained unchanged from baseline levels in others. These and data from other studies suggest that OT responsivity may be an individual difference influenced by factors other than lactation status, and is likely to be modulated by individual and stressor characteristics. For example, in an early series of studies done in healthy adults, plasma OT increased in response to uncontrollable, but not controllable, noise in women but did not increase in men. Importantly OT increased in some, but not all of these female subjects. Those reporting greater tension and feelings of loss of control showed greater increases in OT in response to the stressor. In a separate sample, women scoring high on emotionality also showed significant increases in OT in response to uncontrollable noise (Sanders et al., 1990). More recently Tabak et al. (2011) reported measuring oxytocin reactivity in young women as they described recent betrayals or hurtful interactions in close personal relationships. Greater oxytocin increases were associated with increased postconflict anxiety and decreased levels of forgiveness. The possibility of unexplained, differential OT stress reactivity may complicate human experimental studies because the usual method used to examine OT activity involves insertion of a needle, which is stressful for many people. It also provides additional justification for seeking confirmation of the utility and validity of salivary OT and/or urinary OT measures, which can be obtained with minimally stressful procedures both in the lab and the home environment (Grewen et al., 2010; White-Traut et al., 2009). Urinary oxytocin or a composite measure such as area under the curve, created from multiple saliva or plasma samples, may more closely reflect typical peripheral levels of exposure since oxytocin appears to change rapidly and differently based on subject and task characteristics.

In sum, we have previously reported correlations between higher resting oxytocin and lower resting norepinephrine, heart rate and blood pressure in premenopausal women (Grewen et al., 2005), and between higher levels of an oxytocin precursor and lesser stress-induced vasoconstriction in postmenopausal women (Light et al., 2005a,b). However, this is the first investigation in which plasma oxytocin was linked to both lower norepinephrine and to lesser vasoconstriction and heart rate responses during stress. The present findings are restricted to reproductive age women during the first 6 months after giving birth, and additional studies in larger and broader samples of women and men are needed to confirm 
that the same associations occur in other groups. Still, in their recent warm touch enhancement intervention study, Holt-Lunstad et al. (2008) observed an association in husbands between increased salivary OT and both lower $24 \mathrm{~h}$ BP and sympathetic activity as indexed by salivary alpha amylase, encouraging the interpretation that the present findings may apply to men as well as women.

\section{Acknowledgments}

The authors are grateful to Janet Amico for assay of plasma oxytocin, to Cheryl Walker for assay of prolactin, to Nicole White for assay of norepinephrine, and to Chihiro Christmas for sample processing. This research was funded by NIH grants R01 HL64927, K01DA099949, P01DA022446 and RR00046.

\section{References}

Altemus M, Redwine LS, Leong YM, Frye CA, Porges SW, Carter CS. Responses to laboratory psychosocial stress in postpartum women. Psychosomatic Medicine. 2001; 63(5):814-821. [PubMed: 11573030]

Archer TL, Knape K, Liles D, Wheeler AS, Carter B. The hemodynamics of oxytocin and other vasoactive agents during neuraxial anesthesia for cesarean delivery: findings in six cases. International Journal of Obstetrics Anesthesia. 2008; 17(3):247-254.

Bernatova I, Rigatto KV, Key MP, Morris M. Stress-induced pressor and corticosterone responses in oxytocin-deficient mice. Experimental Physiology. 2004; 89(5):549-557. [PubMed: 15184356]

Callahan MF, Kirby RF, Cunningham JT, Eskridge-Sloop SL, Johnson AK, McCarty R, et al. Central oxytocin systems may mediate a cardiovascular response to acute stress in rats. American Journal of Physiology. 1989; 256(5 Pt 2):H1369-H1377. [PubMed: 2719134]

Cyranowski JM, Hofkens TL, Frank E, Seltman H, Cai HM, Amico JA. Evidence of dysregulated peripheral oxytocin release among depressed women. Psychosomatic Medicine. 2008; 70(9):967975. [PubMed: 19005082]

Grewen KM, Girdler SS, Amico J, Light KC. Effects of partner support on resting oxytocin, cortisol, norepinephrine, and blood pressure before and after warm partner contact. Psychosomatic Medicine. 2005; 67(4):531-538. [PubMed: 16046364]

Grewen KM, Davenport RE, Light KC. An investigation of plasma and salivary oxytocin responses in breast- and formula-feeding mothers of infants. Psychophysiology. 2010; 47(4):625-632. [PubMed: 20102537]

Grippo AJ, Trahanas DM, Zimmerman RR 2nd, Porges SW, Carter CS. Oxytocin protects against negative behavioral and autonomic consequences of long-term social isolation. Psychoneuroendocrinology. 2009; 34(10):1542-1553. [PubMed: 19553027]

Gutkowska J, Jankowski M, Mukaddam-Daher S, McCann SM. Oxytocin is a cardiovascular hormone. Brazilian Journal of Medical and Biological Research. 2000; 33(6):625-633. [PubMed: 10829090]

Heinrichs M, Meinlschmidt G, Neumann I, Wagner S, Kirschbaum C, Ehlert U, et al. Effects of suckling on hypothalamic-pituitary-adrenal axis responses to psychosocial stress in postpartum lactating women. Journal of Clinical Endocrinology and Metabolism. 2001; 86(10):4798-4804. [PubMed: 11600543]

Hill DW, Merrifield AJ. Left ventricular ejection and the Heather Index measured by non-invasive methods during postural changes in man. Acta Anaesthesiologica Scandinavica. 1976; 20(4):313320. [PubMed: 998150]

Holst S, Uvnas-Moberg K, Petersson M. Postnatal oxytocin treatment and postnatal stroking of rats reduce blood pressure in adulthood. Autonomic Neuroscience. 2002; 99(2):85-90. [PubMed: 12241092]

Holt-Lunstad J, Birmingham WA, Light KC. Influence of a "warm touch" support enhancement intervention among married couples on ambulatory blood pressure, oxytocin, alpha amylase, and cortisol. Psychosomatic Medicine. 2008; 70(9):976-985. [PubMed: 18842740]

Jankowski M, Hajjar F, Kawas SA, Mukaddam-Daher S, Hoffman G, McCann SM, et al. Rat heart: a site of oxytocin production and action. Proceedings of the National Academy of Sciences U.S.A. 1998; 95(24):14558-14563. 
Jankowski M, Wang D, Hajjar F, Mukaddam-Daher S, McCann SM, Gutkowska J. Oxytocin and its receptors are synthesized in the rat vasculature. Proceedings of the National Academy of Sciences U.S.A. 2000; 97(11):6207-6211.

Knox SS, Uvnas-Moberg K. Social isolation and cardiovascular disease: an atherosclerotic pathway? Psychoneuroendocrinology. 1998; 23(8):877-890. [PubMed: 9924742]

Kubicek WG, From AH, Patterson RP, Witsoe DA, Castaneda A, Lillehei RC, et al. Impedance cardiography as a noninvasive means to monitor cardiac function. Journal of Association of Advanced Medical Instrumentation. 1970; 4(2):79-84.

Light KC, Smith TE, Johns JM, Brownley KA, Hofheimer JA, Amico JA. Oxytocin responsivity in mothers of infants: a preliminary study of relationships with blood pressure during laboratory stress and normal ambulatory activity. Health Psychology. 2000; 19(6):560-567. [PubMed: 11129359]

Light KC, Grewen KM, Amico JA, Boccia M, Brownley KA, Johns JM. Deficits in plasma oxytocin responses and increased negative affect, stress, and blood pressure in mothers with cocaine exposure during pregnancy. Addictive Behaviour. 2004; 29(8):1541-1564.

Light KC, Grewen KM, Amico JA. More frequent partner hugs and higher oxytocin levels are linked to lower blood pressure and heart rate in premenopausal women. Biological Psychology. 2005a; 69(1):5-21. [PubMed: 15740822]

Light KC, Grewen KM, Amico JA, Brownley KA, West SG, Hinderliter AL, et al. Oxytocinergic activity is linked to lower blood pressure and vascular resistance during stress in postmenopausal women on estrogen replacement. Hormones and Behavior. 2005b; 47(5):540-548. [PubMed: 15811355]

Meisenbert G, Simmons W. Centrally mediated effects of neurohypophseal hormones. Neuroscience Biobehavioral Review. 1983; 7:263-280.

Mezzacappa ES, Kelsey RM, Katkin ES. The effects of epinephrine administration on impedance cardiographic measures of cardiovascular function. International Journal of Psychophysiology. 1999; 31(3):189-196. [PubMed: 10076773]

Michelini LC, Marcelo MC, Amico J, Morris M. Oxytocinergic regulation of cardiovascular function: studies in oxytocin-deficient mice. American Journal of Physiology Heart and Circulatory Physiology. 2003; 284(6):H2269-H2276. [PubMed: 12531722]

Mukaddam-Daher S, Yin YL, Roy J, Gutkowska J, Cardinal R. Negative inotropic and chronotropic effects of oxytocin. Hypertension. 2001; 38(2):292-296. [PubMed: 11509492]

Nakamura H, Itakuara A, Okamura M, Ito M, Iwase A, Nakanishi Y, et al. Oxytocin stimulates the translocation of oxytocinase of human vascular endothelial cells via activation of oxytocin receptors. Endocrinology. 2000; 14(12):4481-4485. [PubMed: 11108258]

Petersson M. Cardiovascular effects of oxytocin. Progressive Brain Research. 2002; 139:281-288.

Petersson M, Uvnas-Moberg K. Effects of an acute stressor on blood pressure and heart rate in rats pretreated with intracerebroventricular oxytocin injections. Psychoneuroendocrinology. 2007; 32(8-10):959-965. [PubMed: 17698297]

Petersson M, Uvnas-Moberg K. Postnatal oxytocin treatment of spontaneously hypertensive male rats decreases blood pressure and body weight in adulthood. Neuroscience Letters. 2008; 440(2):166169. [PubMed: 18562099]

Petersson M, Alster P, Lundeberg T, Uvnas-Moberg K. Oxytocin causes a long-term decrease of blood pressure in female and male rats. Physiology and Behavior. 1996; 60(5):1311-1315. [PubMed: 8916187]

Petersson M, Lundeberg T, Uvnas-Moberg K. Oxytocin decreases blood pressure in male but not in female spontaneously hypertensive rats. Journal of Autonomic Nervous System. 1997; 66(1-2): 15-18.

Petersson M, Lundeberg T, Uvnas-Moberg K. Oxytocin enhances the effects of clonidine on blood pressure and locomotor activity in rats. Journal of Autonomic Nervous System. 1999a; 78(1):4956.

Petersson M, Lundeberg T, Uvnas-Moberg K. Short-term increase and long-term decrease of blood pressure in response to oxytocin-potentiating effect of female steroid hormones. Journal of Cardiovascular Pharmacology. 1999b; 33(1):102-108. [PubMed: 9890403] 
Pierrehumbert B, Torrisi R, Laufer D, Halfon O, Ansermet F, Beck Popovic M. Oxytocin response to an experimental psychosocial challenge in adults exposed to traumatic experiences during childhood or adolescence. Neuroscience. 2010; 166(1):168-177. [PubMed: 20018229]

Pruessner J, Kirschbaum C, Meinlschmid G, Hellhammer D. Two formulas for computation of the area under the curve represent measures of total hormone concentration versus time-dependent change. Psychoneuroendocrinology. 2003; 28:916-931. [PubMed: 12892658]

Rosen, M. Rosen Listening Touch. North Atlantic Books; Berkeley, CA: 2003.

Sanders G, Freilicher J, Lightman SL. Psychological stress of exposure to uncontrollable noise increases plasma oxytocin in high emotionality women. Psychoneuroendocrinology. 1990; 15(1): 47-58. [PubMed: 2367615]

Sartain JB, Barry JJ, Howat PW, McCormack DI, Bryant M. Intravenous oxytocin bolus of 2 units is superior to 5 units during elective Caesarean section. British Journal of Anaesthesia. 2008; 101(6): 822-826. [PubMed: 18845650]

Sherwood A, Allen MT, Fahrenberg J, Kelsey RM, Lovallo WR, van Doornen LJ. Methodological guidelines for impedance cardiography. Psychophysiology. 1990a; 27(1):1-23. [PubMed: 2187214]

Sherwood A, Dolan CA, Light KC. Hemodynamics of blood pressure responses during active and passive coping. Psychophysiology. 1990b; 27(6):656-668. [PubMed: 2100351]

Simpson KR, Knox GE. Oxytocin as a high-alert medication: implications for perinatal patient safety. MCN American Journal of Maternal and Child Nursing. 2009; 34(1):8-15. quiz 16-17.

Sofroniew M. Vasopressin and oxytocin in the mammalian brain and spinal cord. Trends in Neuroscience. 1983; 6:467-472.

Stock S, Uvnas-Moberg K. Increased plasma levels of oxytocin in response to electrical stimulation of the sciatic and vagal nerves in anesthetized rats. Acta Physiologica Scandinavica. 1988; 132:2934. [PubMed: 3223304]

Szeto A, Nation DA, Mendez AJ, Dominguez-Bendala J, Brooks LG, Schneiderman N, et al. Oxytocin attenuates NADPH-dependent superoxide activity and IL-6 secretion in macrophages and vascular cells. American Journal of Physiology, Endocrinology and Metabolism. 2008; 295(6):E1495E1501. [PubMed: 18940936]

Tabak BA, McCullough ME, Szeto A, Mendez AJ, McCabe PM. Oxytocin indexes relational distress following interpersonal harms in women. Psychoneuroendocrinology. 2011; 36(1):115-122. [PubMed: 20688437]

Taylor SE, Gonzaga GC, Klein LC, Hu P, Greendale GA, Seeman TE. Relation of oxytocin to psychological stress responses and hypothalamic-pituitary-adrenocortical axis activity in older women. Psychosomatic Medicine. 2006; 68(2):238-245. [PubMed: 16554389]

Taylor SE, Saphire-Bernstein S, Seeman TE. Are plasma oxytocin in women and plasma vasopressin in men biomarkers of distressed pair-bond relationships? Psychological Science. 2010; 21(1):3-7. [PubMed: 20424014]

Thibonnier M, Conarty D, Preston J, Plesnicher C, Dweik R, Erzurum S. Human vascular endothelial cells express oxytocin receptors. Endocrinology. 1999a; 140(3):1301-1309. [PubMed: 10067857]

Thibonnier M, Conarty DM, Preston JA, Plesnicher CL, Dweik RA, Erzurum SC. Human vascular endothelial cells express oxytocin receptors. Endocrinology. 1999b; 140(3):1301-1309. [PubMed: 10067857]

Thomas JS, Koh SH, Cooper GM. Haemodynamic effects of oxytocin given as i.v. bolus or infusion on women undergoing Caesarean section. British Journal of Anaesthesia. 2007; 98(1):116-119. [PubMed: 17142825]

Turner RA, Altemus M, Enos T, Cooper B, McGuinness T. Preliminary research on plasma oxytocin in normal cycling women: investigating emotion and interpersonal distress. Psychiatry. 1999; 62(2):97-113. [PubMed: 10420425]

Uvnas-Moberg K. Oxytocin may mediate the benefits of positive social interaction and emotions. Psychoneuroendocrinology. 1998; 23(8):819-835. [PubMed: 9924739]

Uvnas-Moberg K, Petersson M. Oxytocin-biochemical link for human relations. Mediator of antistress, well-being, social interaction, growth, healing. Lakartidningen. 2004; 101(35):26342639. [PubMed: 15458215] 
Uvnas-Moberg K, Johansson B, Lupoli B, Svennersten-Sjaunja K. Oxytocin facilitates behavioural, metabolic and physiological adaptations during lactation. Applied Animal Behavioral Science. 2001; 72(3):225-234.

White-Traut R, Watanabe K, Pournajafi-Nazarloo H, Schwertz D, Bell A, Carter CS. Detection of salivary oxytocin levels in lactating women. Developmental Psychobiology. 2009; 51(4):367-373. [PubMed: 19365797]

Wsol A, Cudnoch-Jedrzejewska A, Szczepanska-Sadowska E, Kowalewski S, Puchalska L. Oxytocin in the cardiovascular responses to stress. Journal of Physiology and Pharmacology. 2008; 59(Suppl. 8):123-127. [PubMed: 19258669] 

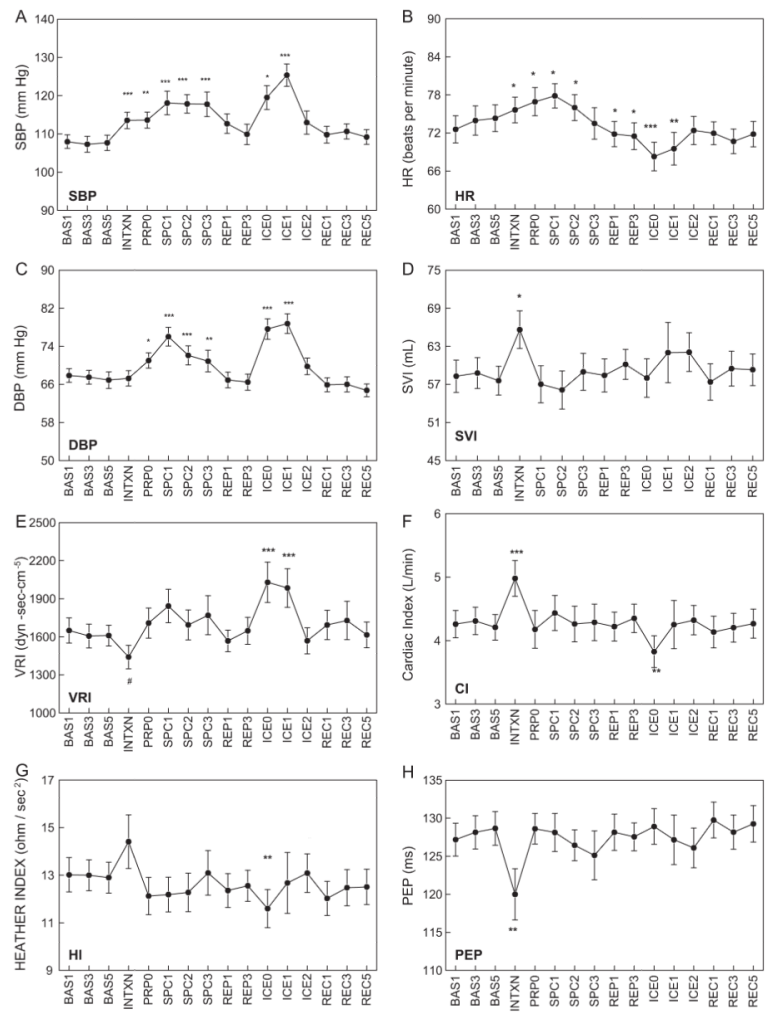

Fig. 1.

Mean cardiovascular values at each recording, displayed as mean \pm standard error of the mean. Asterisks identify values that are significantly different than the mean of resting baseline values recorded at Minutes 1, 3, and 5 (BAS1, BAS3, BAS5). INTXN: infant interaction; PRP0: onset of speech preparation; SPC: speech; REP: speech replay; ICE: cold pressor; REC: recovery. SVI: stroke volume index; VRI: vascular resistance index; CI: cardiac output index; HI: heather index; PEP: pre-ejection period; ${ }^{\#} p<0.10,{ }^{*} p<0.05$, ${ }^{* *} p$ $<0.01, * * * p<0.001$. 

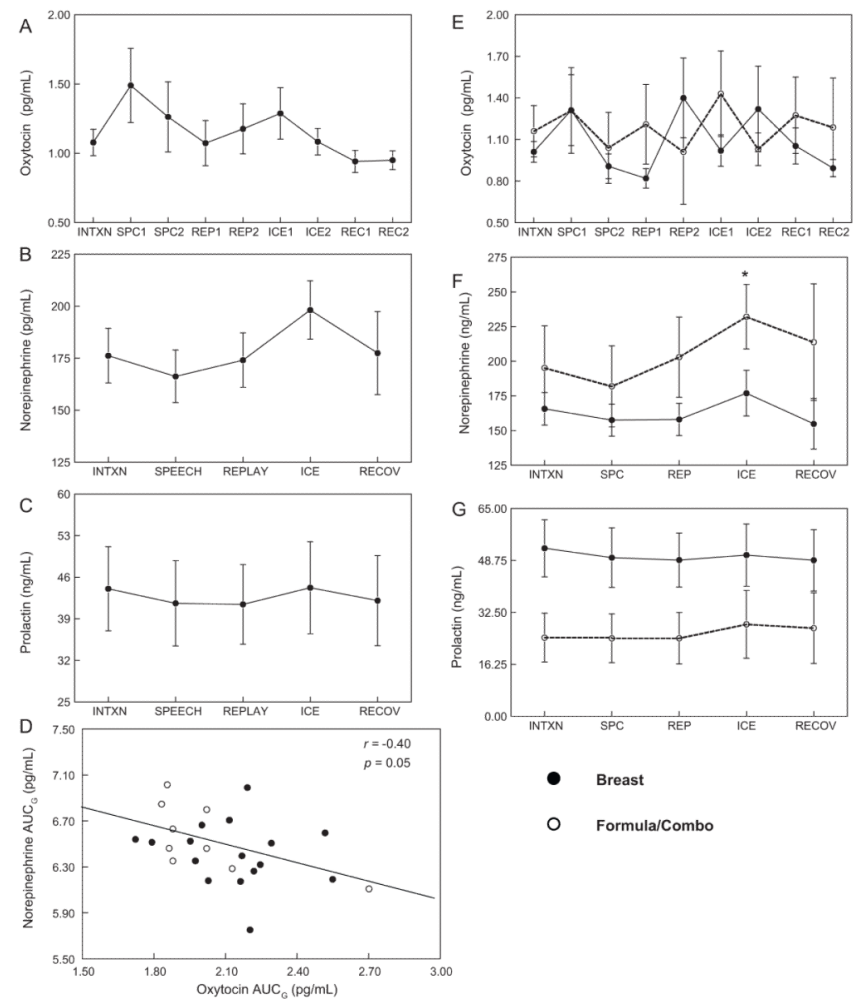

- Breast

O Formula/Combo

Fig. 2.

Values for plasma oxytocin (A), norepinephrine (B), and serum prolactin (C), displayed as mean \pm standard error of the mean at each measurement. (D) Scatterplot and regression line reflecting Pearson correlation $(r)$ of $\log$ Norepinephrine $\mathrm{AUG}_{\mathrm{G}}$ and $\log$ Oxytocin $\mathrm{AUC}_{\mathrm{G}}$. Values in plasma based on infant feeding method (18 breast, 10 formula in whole or in combination with breast) for: (E) oxytocin, (F) norepinephrine, and (G) serum prolactin. 

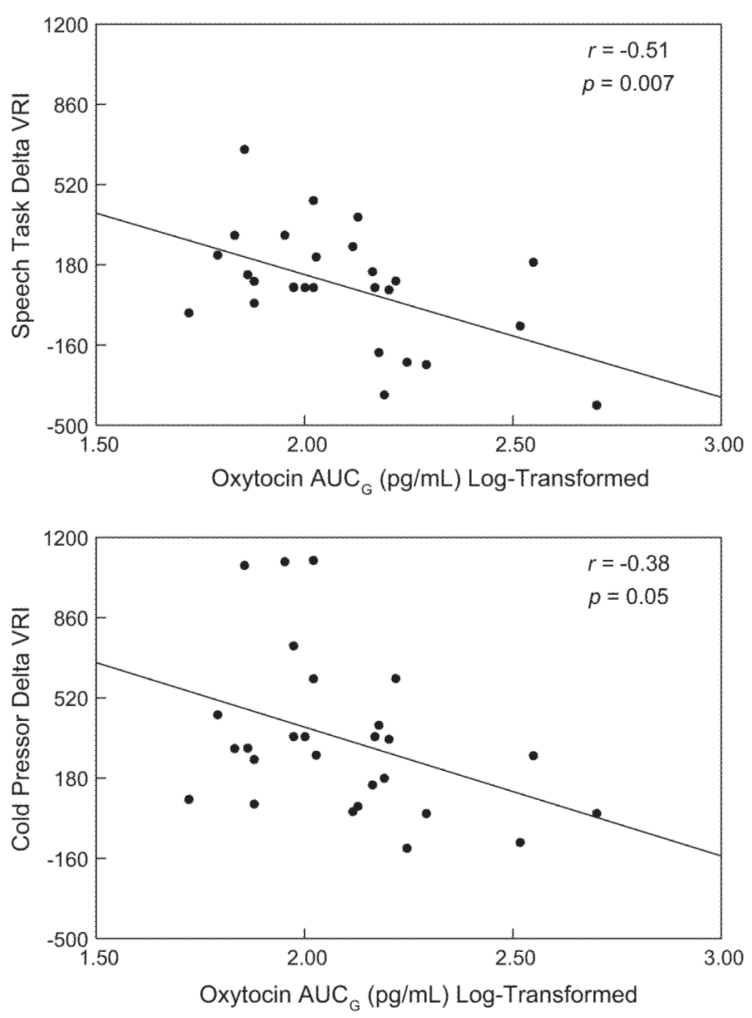

Fig. 3.

(A) Scatterplot and regression lines reflecting Pearson correlations $(r)$ between delta VRI (dyn-s-cm ${ }^{5}$ ) during speech (speech Minute 1 - mean baseline) with log Oxytocin AUC $\mathrm{G}_{\mathrm{G}}$ (B) Scatterplot and regression lines reflecting Pearson correlation between delta VRI during cold pressor (ICE) with log oxytocin $\mathrm{AUC}_{\mathrm{G}}$. 


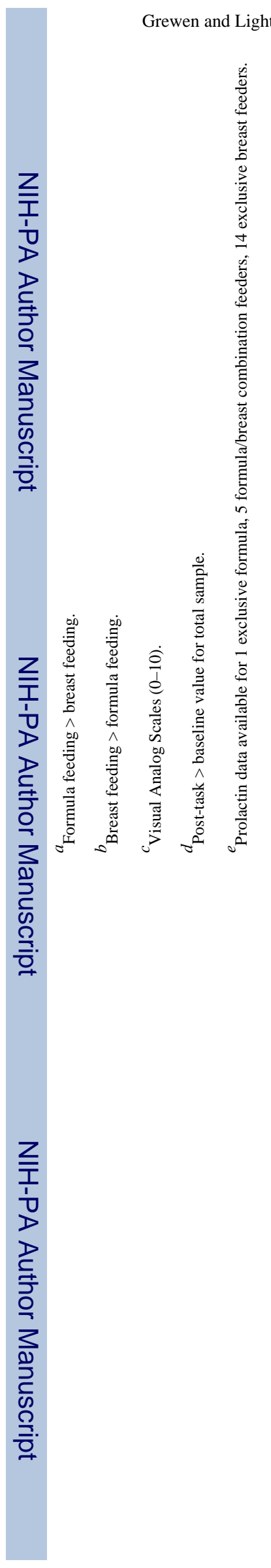

Page 20

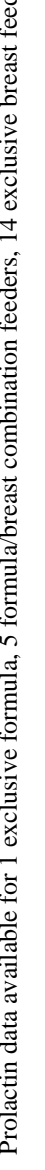

.




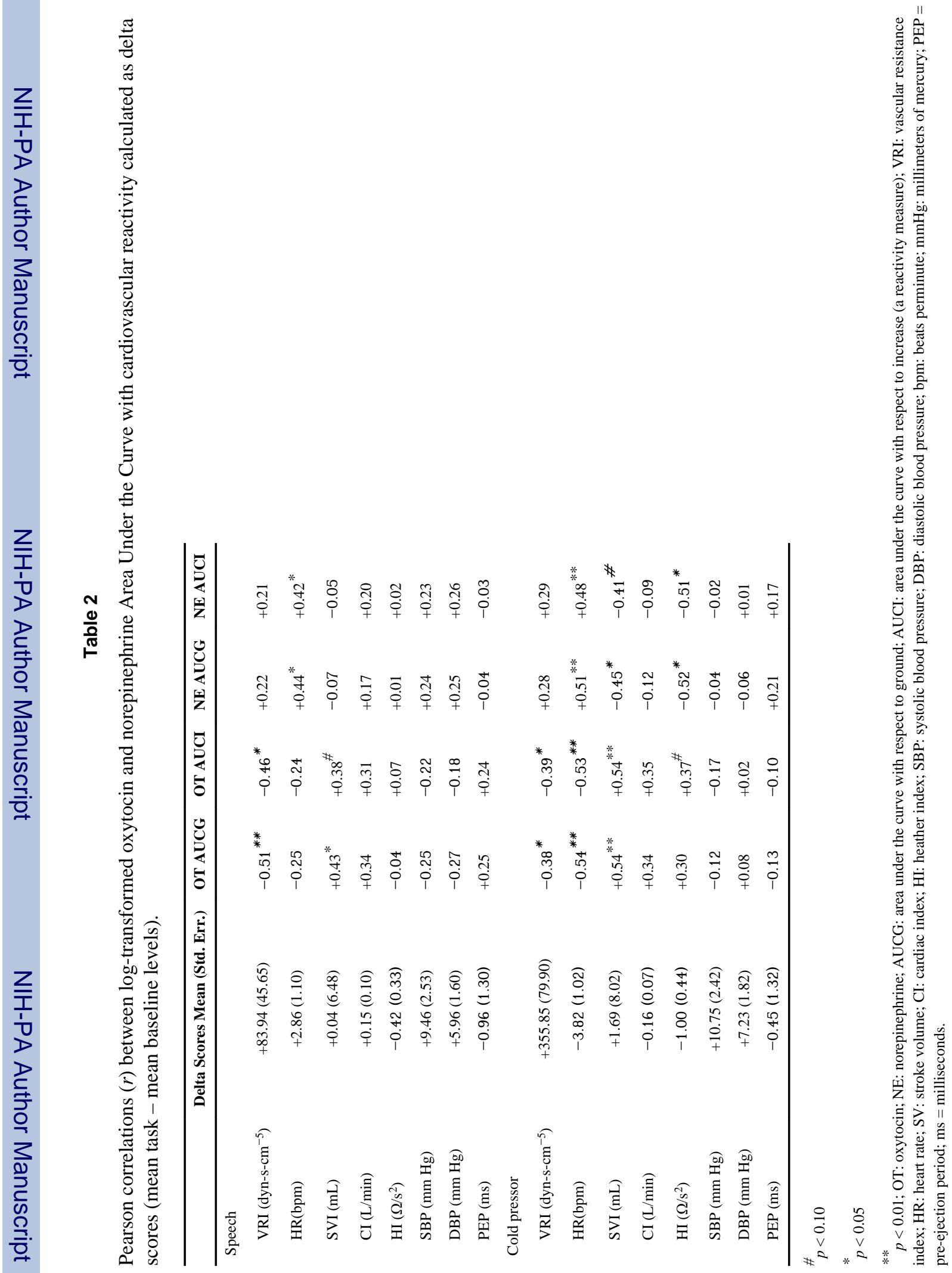

